\title{
Essay Review: Complexity Features, (Putative) Truisms, and the Ising Model
}

\author{
James Ladyman \& Karoline Wiesner: What Is a Complex System? \\ Yale University Press, New Haven and London 2020, 184 pp., \$35.00 \\ (Paperback), ISBN: 9780300251104
}

\section{Tobias Henschen ${ }^{1}$}

Accepted: 11 January 2021 / Published online: 29 April 2021

(C) The Author(s) 2021

In the preface to their relatively short (169-page) book, Ladyman and Wiesner (LW, henceforth) state that it aims to undertake "a thorough analysis" which would answer questions such as "Is complexity a truly new phenomenon or merely a new label?"; "Can the different conceptions of physicists, biologists, social scientists and others be brought into a single framework, or do they address different and unrelated phenomena?"; "Are measures of complexity meaningful for a phenomenon this multi-faceted?"; and "Why are information theory and network theory so prominent in complexity science?" (p. ix). The analysis is meant to be thorough in the sense of defining "precisely the terms of the debate, the phenomena they describe, and the relations between these phenomena" (p. ix).

LW believe that such definitions "would be beneficial" because "the field of complexity sciences has harbored a lot of confusion", "[1]ack of clarity [...] is detrimental to science", and "confused foundations are sometimes highly problematic" (p. ix). LW do not provide a lot of evidence in support of the confusion, incompatibilities, and non-sequiturs that they say characterize the debate (pp. ix, 13, 77). Everyone familiar with the debate, however, will probably know what they have in mind. And their book should indeed be viewed as an important contribution to a more precise understanding of the terms of the debate, the phenomena they describe and the relations between them. In this short essay, I will take this view for largely granted. I will substantiate it only briefly and dwell, for the most part, on a number of points which I think deserve critical discussion. I will begin by briefly summarizing its five chapters.

Chapter 1 (the introduction) contains a statement of what LW take to be 9 "truisms of complexity science", a list of 10 features that LW believe are associated with complex systems (Sect. 1.1) and a brief historical account of complexity science (Sect. 1.2). The "truisms of complexity science" include statements such as "more is different", "nonliving systems can generate order", "complexity can come from simplicity" and "coordinated behavior does not require an overall controller". LW say of these "truisms" that they "will not be obvious to everyone", but that they will be obvious "to those working

Tobias Henschen

tobias.henschen@uni-koeln.de

1 Philosophy Department, University of Cologne, Aachener Straße 217, 50931 Cologne, Germany 
in complexity science" (p. 9). They also say, however, that the following "truisms" "are the least obvious and most in need of articulation and argument" (p. 9): "There are various kinds of invariance and forms of universal behavior in complex systems" (truism 6) and "There is a difference between the order that complex systems produce and the order of complex systems themselves" (truism 9). The brief historical account covers well-known developments in cybernetics, dynamical systems theory and cellular automata research as well as research undertaken at the Los Alamos National Laboratory and Santa Fe Institute to understand the more general features of complex systems.

Chapter 2 reviews typical examples of complex systems. That review is meant to emphasize the diversity of these systems, their commonalities, and the wide range of features that they display (p. x). The complex systems reviewed are either nonliving or living. The nonliving systems include systems studied by thermal physics, the universe, our solar system and the climate. The living systems are either systems of human construction or unconstructed systems. The systems of human construction comprise the economy and the World Wide Web, the unconstructed systems eusocial insects and the human brain.

Chapter 3 analyzes the 10 features of complex systems listed in the introduction. They run as follows (cf. pp. 65-6):

(1) Numerosity: complex systems involve many interactions among many components.

(2) Disorder and diversity: the interactions in a complex system are not coordinated or controlled centrally, and the components may differ.

(3) Feedback: the interactions in a complex system are iterated so that there is feedback from previous interactions on a time scale relevant to the system's emergent dynamics.

(4) Non-equilibrium: complex systems are open to the environment and are often driven by something external.

(5) Spontaneous order and self-organization: complex systems exhibit structure and order that arises out of the interactions among their parts.

(6) Non-linearity: complex systems exhibit nonlinear dependence on parameters or external drivers.

(7) Robustness: the structure and function of complex systems is stable under relevant perturbations.

(8) Nested structure and modularity: there may be multiple scales of structure, clustering and specialization of function in complex systems.

(9) History and memory: complex systems often require a very long history to exist and often store information about history.

(10) Adaptive behavior: complex systems are often able to modify their behavior depending on the state of the environment and the predictions they make about it.

LW say that "[n]ot all these features are present in all complex systems" (p. 66), that "not all the products are found in all complex systems" (p. 66), and that "[o]ften products help produce other products" (p. 66). Diversity, for instance, is a feature that is not present in all complex systems (p. 70). Modularity, memory, and adaptivity cannot be found in complex nonliving systems (p. 131). And adaptivity helps produce other products like modularity (p. 66).

One specific feature that many theorists believe is a necessary condition for the complexity of a system is perspicuously absent from the list: emergence. In fact, however, emergence is not really absent. LW say that the "interesting thing about complex systems" (p. 65) is that (1) to (4) represent "conditions" that can give rise to "products" (5) to (10). 
And in a move that is interesting and novel they say that the conditions give rise to the products in the sense that the latter "emerge" from the former (cf. especially Sect. 3.5). But there is another feature that is perspicuously absent from the list: sensitivity to initial conditions ("chaos"). This is the feature that many physicists and engineers mention first when asked to state features of complex systems. LW (pp. 78, 100) state two reasons why chaos is neither necessary nor sufficient for complexity. The first reason is that chaos is a special feature of some deterministic systems, and that many complex systems are nondeterministic (or stochastic). The second reason is that some chaotic systems (e.g. the chaotic pendulum) are non-complex.

Chapter 4 relates the ten features to measures of complexity used by scientists. Numerosity is related to a simple count of entities and interactions between them (p. 88); disorder to variance, understood as the expectation of the squared deviation of a random variable from its mean (p. 89), or Shannon entropy (i.e. the information-theoretic measure of the amount of uncertainty in a probability distribution; p. 90); diversity to Shannon entropy (p. 92); non-equilibrium to the time-independent probability of a system transitioning from one state to another (pp. 95-6); order to covariance, understood as a standard measure of correlation (p. 96), or mutual information (i.e. a function of Shannon entropy that may likewise be taken to measure correlation; p. 97); nonlinearity to the variance of a power-law distribution if nonlinearity is understood as power-law behavior (pp. 99-100), or to covariance or mutual information if nonlinearity is taken to be synonymous with correlation ( $\mathrm{p}$. $101)$; robustness to the number of negative real parts of the eigenvalues of the Jacobian matrix of a system evaluated at a given fixed point (p. 102), to the time it takes for a system to return to a steady state after a perturbation (p. 103), or to the variance of a power-law distribution if the system is driven towards a critical point (p. 105); nested structure to fractal dimension (pp. 108-9); modularity to clustering in networks (p. 107); and memory to correlations in time (p. 110).

LW point out that there is no measure of feedback, self-organization or history as such, but that measures of the effects of feedback (such as order and nonlinearity) can be regarded as indicators for feedback (p. 93), that measures of order can be understood as proxies for self-organization if specific background knowledge is available (p. 96), and that almost any complexity measure can be used as proxy for history because all features of complex systems arise because of their histories (p. 110). Chapter 4 also discusses complexity measures that LW refer to as "classic": complexity measures that they say "do not belong in a practitioner's tool kit", because they "were constructed as thought experiments rather than as measures to be applied to real-world systems" (p. 88). These measures include thermodynamic depth, statistical complexity, effective complexity and logical depth (cf. Section 4.10).

Chapter 5 provides answers to the questions posed in the preface. These answers state that "complexity is not just a label", but "not a single phenomenon" either; that the "different conceptions of complexity of physicists, biologists, social scientists and others cannot be brought into a single framework", but that "there are multiple frameworks, such as information theory, network theory, and the theory of critical phenomena, that can be applied across the sciences"; and that "[m]easures of complexity are meaningful but measure different features of complex systems that manifest themselves in different ways" ( $\mathrm{p}$. 130).

If complexity is not a single phenomenon, one may wonder about the position that one would have to adopt with respect to the ontological status of complex systems. LW consider three such positions. According to the first ("nihilism"), there is no such thing as (an essence of) complexity or complex systems (p. 117). The second position ("pragmatism") 
remains agnostic about whether there is such an essence (p. 119). The third position ("realism") affirms that there is such an essence: that complex systems form a natural kind (p. 120). LW choose to adopt the realist position. And they say that in order to defend that position, they just need to be "able to say something, vague or not, about what complex systems are" (p. 120).

What is it that they say about what complex systems are? They do not wish to endorse generic conceptions (conceptions that can be stated using the language of dynamical systems theory), physical conceptions (conceptions that can be stated using only the language of physics), computational conceptions (conceptions that can be stated using only information-theoretic vocabulary), or functional conceptions (conceptions that are restricted to living systems or systems displaying adaptive behavior) of complexity. They say, instead, that "[t]he 'conditions' for complexity—numerosity, disorder and diversity, feedback, and non-equilibrium—give rise to the 'products' of order and organization, robustness, and nonlinearity", that "[s]ystems with all the features of order, robustness, nonlinearity, nested structure and history are at the highest level of complexity found in systems that are not ascribed functions", and that "adaptive behavior leads to the functional features of forms of self-organization and order, modularity, and memory", once function is involved (p. 130).

Chapter 5 ends by briefly considering ethical and political implications of complexity science and is followed up by an appendix, which defines the mathematical terminology used in chapter 4 more rigorously and presents the basics of probability theory, information theory, algorithmic complexity, and network theory.

The major strength of the book is that it achieves its aim of rendering our understanding of the terms of the debate, the phenomena they describe and the relations between them more precise. As far as I can see, it is by essentially three important moves that the book achieves this aim. The first move is that of parallelizing features of complex systems and their respective measures. Many of these features-order, for instance-are notoriously difficult to understand. But when supplemented with a measure, they become very easy to understand. If order (disorder) is measured by covariance or mutual information (variance or Shannon entropy), then order (disorder) is just the presence (absence) of correlation.

The second move is that of interpreting emergence as "emergence" of "products" (5) to (10) from "conditions" (1) to (4). Emergence is a feature that is likewise notoriously difficult to understand, even though it has been at the center of interest of theorists at least since the early days of the Santa Fe Institute. To my mind, the interpretation of emergence as emergence of products from conditions is a lot clearer and a lot more relevant to science than competing interpretations of emergence in terms of irreducibility, unpredictability, unexplainability, ontological novelty, supervenience, and so on.

The third move is that of distinguishing the different senses, in which scientists and philosophers use the term "nonlinearity", and of then saying exactly, in which of these senses nonlinearity is or is not a necessary or sufficient condition for the complexity of a system. If nonlinearity is a property of systems of equations (i.e. of equations that fail to satisfy superposition or homogeneity), then nonlinearity is neither necessary nor sufficient for the complexity of a system (p. 78). If "nonlinearity" means as much as "correlation" (p. 101), then nonlinearity is a necessary, but not sufficient condition for the complexity of a system (because correlation measures order, and because order is a necessary but not sufficient condition for complexity, cf. p. 77). If nonlinearity is a feature "on the level of the whole" (p. 78), then nonlinearity is likewise a necessary, but not sufficient condition for the complexity of a system (then the system is not a linear sum of its parts): then "what the individuals do together is different from the sum of what each one of them does alone" (p. 78). 
The major strength of the book easily outweighs all of its weaknesses and makes it an important read for all of the target groups mentioned in the preface: "students and academics interested in complexity science and the nature of complexity", "scientific practitioners in related areas", and "scientifically informed general readers" (p. ix). It is these weaknesses, however, that I will be concerned with (on a scale from less to more serious) in the remainder of this short essay. The first weakness relates to difficulties of reading or studying the text. The book's relative shortness, its lack of engagement with the "extensive philosophical literature" (p. x), its intended audience, and the addition of an appendix suggest a somewhat easy read. But a complete lack of figures and gaps in the analysis turn the book into an unnecessarily difficult read that only the most eager students and general readers will undergo. In the literature on complex systems, figures are often employed to illustrate phase transitions, critical points, thermohaline circulation, the equilibration of prices, financial market crashes and so on. But the only figure appearing in the book is a fancy illustration of the features of a complex system and their interrelations (p. 131).

A typical gap in the analysis is the failure to show that the 9 "truisms" are true of the study of the complex systems reviewed in chapter 2. LW claim that "[a]ll the truisms of complexity science [...] are true of the study of nonliving matter and radiation" (p. 28). And they substantiate that claim for all "truisms" (except "truism" 5). But they fail to substantiate corresponding claims for the other complex systems reviewed in chapter 2. Another gap is the occasional lack of integration between the complexity features listed in chapter 3 and the complexity measures discussed in chapter 4 . The interpretation of correlation as a measure of nonlinearity in 4.6 .3 comes as a surprise because the earlier Sect. 3.7 does not contain anything that allows the reader to anticipate that interpretation. For some reason, LW do not present a measure of adaptivity: they present measures or proxies for all other features, but don't loose a word about why a measure or proxy is missing in the case of adaptivity.

One final gap relates to the somewhat enigmatic statement that nonlinearity is "not sufficient for a complex system (on any but the most all-encompassing conception)" (p. 78). What is the most all-encompassing conception of nonlinearity? It would be very nice for LW to be able to revert to such a conception, because (as pointed out above) they wish to endorse a realist position with respect to complex systems. They fail to explain, however, under what conception nonlinearity amounts to a sufficient condition for the complexity of a system. They state that "[i]n the popular and philosophical literature on complex systems a lot of heat and little light is often generated by talk of linearity and nonlinearity" (p. 77). While this statement is probably true, one might want to point out that LW's talk of nonlinearity is not exceptional in the sense that it produces only light.

A second weakness of the book pertains to the epistemic status of the 9 "truisms" listed in the preface. If statements (6) and (9) represent "truisms", then why are they "least obvious and most in need of articulation and argument" (p. 9)? It seems to me that statement (6) that there are "various kinds of invariance and forms of universal behavior in complex systems" comes close to a truism because it is widely agreed and doesn't seem to need a lot of articulation and argument. It might be difficult to decide just when the details of the physical interactions between the parts of a system can be ignored in favor of invariance or universal behavior. But that is a decision that practicing scientists need to make. Hardly any philosopher or scientist doubts that the details of the physical interactions between the parts of a system can be ignored in many cases. LW cite at least one such case (p. 85): the case of the swarming behavior of insects that exchange information-a case, in which it doesn't matter whether the insects exchange information (or 'signal' to each other) by scent, sight, or sound. 
I do not believe, however, that statement (9) that there is "a difference between the order that complex systems produce and the order of complex systems themselves" comes close to a truism. Toward the end of this short essay, I am going to discuss the example of a class of complex systems, the order of which cannot be easily distinguished from the order that these systems produce. I also think that the presence of such systems indicates that scientific practice determines the epistemic status of "truisms" like statement (9), and that in scientific practice, there are not any (long-lived) truisms.

A third weakness of the book relates to the position of realism that LW wish to endorse with respect to complex systems. They are wrong when suggesting that in order to defend that position, they just need to be "able to say something, vague or not, about what complex systems are" (p. 120). In order to defend that position, they need to state necessary and sufficient conditions for the complexity of a system and argue convincingly (against conventionalism) that these conditions hold independently of scientific practice. They make a good case for the claim that most of the ten features that they analyze in chapter 3 qualify as necessary conditions for the complexity of a system. But they fail to specify any sufficient conditions: they fail to explain under what conception nonlinearity might qualify as a sufficient condition, and they do not seem to believe that a conjunction of complexity features amounts to a sufficient condition. And they nowhere make a convincing case against conventionalism (or pragmatism, for that matter).

A related and final weakness has to do with the fact that the complexity features listed in chapter 3 might fail to get the rich phenomenology of complex systems fully under control. When reading the book (especially chapters 3 and 4), one feels inevitably drawn to the game of considering specific systems, and of checking whether they exhibit all the complexity features that LW list. I played the game from bottom to top (so to speak), and even at the bottom, I found it difficult to decide whether some of the complexity features apply. In the remainder of this short essay, I want to address some of these difficulties by considering the Ising model (cf. e.g. Bruce and Wallace 1989): a model that statistical physicists use to model a whole (universality) class of systems (ferromagnetism, liquid-vapor mixtures, binary alloys etc.). LW do not consider this model explicitly, but they deal with ferromagnets, phase transitions, critical phenomena, and scaling laws on several occasions (pp. 24-27, 103-106). Many physicists believe that the model represents complex systems, despite its non-chaotic character.

Systems like ferromagnets clearly exhibit numerosity, as they involve a large number of interactions between a large number of system components. They are not diverse because there is only one type of component: sites that are associated with binary variables that attain values under specific arrangements. The systems exhibit disorder in the sense of homogeneity (p. 69) if the so-called order parameter is taken to measure order, and disorder in the sense of lack of correlation (p. 89) if correlation length is taken to measure order. There is the problem that the order parameter is at a minimum if the system is totally nonhomogenous (if +1 is assigned to exactly half the sites), and at a maximum if the system is totally homogenous (if each site is assigned the same value). But the problem relates to a way of speaking and would disappear if we spoke of a 'disorder parameter' instead. The systems exhibit feedback, which is modeled by an algorithm that takes the number assigned to a site under arrangement $a_{n}$ into the number assigned to the same site under arrangement $a_{n+1}$ with a specific probability, which depends on the total interaction energy of arrangement $a_{n}$. Are the systems in thermal and dynamic non-equilibrium? For specific temperatures, the answer is negative. But once temperature changes abruptly and significantly, the systems will be kicked out of equilibrium. 
Do the systems exhibit spontaneous order? Order is lack of disorder and as such either non-homogeneity or correlation. LW say that order is spontaneous if it arises from the aggregate of a very large number of disordered and uncoordinated interactions between elements without external control (p. 96). The systems clearly exhibit order in the sense of maximum non-homogeneity if absolute temperature is at or above the critical temperature, and order in the sense of maximum correlation length if absolute temperature is near the critical temperature. It is not entirely clear, however, whether order can be said to arise spontaneously. The order (or 'disorder') parameter and correlation length are functionally dependent on temperature, and temperature is externally controlled. ${ }^{1}$

The systems are nonlinear in all three senses because the power laws that govern the variation with temperature of quantities like the order parameter and correlation length are expressed by equations that fail to satisfy superposition and homogeneity; because there is coupling strength, which couples sites (or blocks of sites) of various sizes; and because there is interaction energy or the tendency of variables of adjacent sites or blocks to become equal. The systems also have nested structure in the sense that the probability distribution of scaled block variables is a scale-invariant property. And they have a history, which is simulated by a stochastic algorithm that takes the number assigned to a site under arrangement $a_{n}$ into the number assigned to the same site under arrangement $a_{n+1}$, such that repeated applications of the algorithm generate a sequence of numbers assigned to the site under arrangements $a_{0}, a_{1}, a_{2}$ and so on. LW argue that "[s]ome kind of robustness is necessary for any kind of order" (p. 80). Systems like ferromagnets are hardly robust, however, because structure or order in the sense of nested structure, scale invariance, or maximum correlation length is inherently unstable, and because the systems do not return to order in the sense of maximum inhomogeneity unless absolute temperature remains at or rises above the critical temperature. ${ }^{2}$

It is worth noting that the systems exhibit adaptivity if adaptivity is understood (in accordance with an alternative usage of the term that is probably just as common) as the capacity of system components to modify (or adapt) their interactions in response to system behavior (cf. e.g. Rickles 2011, 535). It is true that the systems do not exhibit adaptivity if adaptivity is understood (with LW) as an exclusive feature of living systems. But they exhibit adaptivity in the alternative sense because the power laws that govern the variation with temperature of quantities, like the order parameter and correlation length, determine interactions between system components: because the order that these systems produce (the power laws) likewise produces the order of these systems (that LW say coexists with their disorder, p. 69). The fact that complex systems like ferromagnets, liquid-vapor mixtures and so on exhibit adaptivity in this alternative sense suggests that "truism" 9 is not a truism after all.

In conclusion, let me repeat that I think that the major strength of the book is that it achieves its aim of rendering our understanding of the terms of the debate, the phenomena they describe and the relations between them more precise. Let me emphasize again that I think that the major strength of the book easily outweighs all of its weaknesses. One of these weaknesses concerns certain difficulties of reading or studying the book. The other weaknesses relate to points, which I think merit being pursued further.

\footnotetext{
${ }^{1} \mathrm{LW}$ in fact seem to deny that order is spontaneous in the case of the Ising model. They say that the criticality of ferromagnets is not self-organized because parameters need to be tuned in order to arrive at the critical point (p. 27).

2 LW remark in this context that the "seeming contradiction between the robustness of a complex system [...] and the inherent instability of systems close to a tipping point remains unresolved" (p. 106).
} 
Funding Open Access funding enabled and organized by Projekt DEAL.

Open Access This article is licensed under a Creative Commons Attribution 4.0 International License, which permits use, sharing, adaptation, distribution and reproduction in any medium or format, as long as you give appropriate credit to the original author(s) and the source, provide a link to the Creative Commons licence, and indicate if changes were made. The images or other third party material in this article are included in the article's Creative Commons licence, unless indicated otherwise in a credit line to the material. If material is not included in the article's Creative Commons licence and your intended use is not permitted by statutory regulation or exceeds the permitted use, you will need to obtain permission directly from the copyright holder. To view a copy of this licence, visit http://creativecommons.org/licenses/by/4.0/.

\section{References}

Bruce, A., \& Wallace, D. (1989). Critical point phenomena, universal physics at large length scales. In P. Davies (Ed.), The new physics (pp. 236-267). Cambridge: CUP.

Rickles, D. (2011). Econophysics and the complexity of financial markets. In C. Hooker (Ed.), Philosophy of complex systems (pp. 532-565). Oxford: North Holland.

Publisher's Note Springer Nature remains neutral with regard to jurisdictional claims in published maps and institutional affiliations. 